\title{
Superinfection of Laodelphax striatellus with Wolbachia from Drosophila simulans
}

\author{
L Kang, X Ma, L Cai, S Liao, L Sun, H Zhu, X Chen, D Shen, S Zhao and C Li \\ Institute of Genetics, State Key Laboratory of Genetic Engineering, Fudan University, Shanghai 200433, China
}

\begin{abstract}
Wolbachia are maternally inherited, intracellular $\alpha$-proteobacteria that infect a wide range of arthropods. They manipulate the reproduction of hosts to facilitate their spread into host populations, through ways such as cytoplasmic incompatibility $(\mathrm{Cl})$, parthenogenesis, feminization and male killing. The influence of Wolbachia infection on host populations has attracted considerable interest in their possible role in speciation and as a potential agent of biological control. In this study, we used both microinjection and nested PCR to show that the Wolbachia naturally infecting Drosophila simulans can be transferred into a naturally Wolbachiainfected strain of the small brown planthopper Laodelphax striatellus, with up to $30 \%$ superinfection frequency in the $F_{12}$ generation. The superinfected males of $L$. striatellus showed unidirectional $\mathrm{Cl}$ when mated with the original single-infected females, while superinfected females of $L$. striatellus were
\end{abstract}

compatible with superinfected or single-infected males. These results are, to our knowledge, the first to establish a superinfected horizontal transfer route for Wolbachia between phylogenetically distant insects. The segregation of Wolbachia from superinfected $L$. striatellus was observed during the spreading process, which suggests that Wolbachia could adapt to a phylogenetically distant host with increased infection frequency in the new host population; however, it would take a long time to establish a highfrequency superinfection line. This study implies a novel way to generate insect lines capable of driving desired genes into Wolbachia-infected populations to start population replacement.

Heredity (2003) 90, 71-76. doi:10.1038/sj.hdy.6800180

Keywords: Wolbachia; microinjection; Laodelphax striatellus; Drosophila simulans; unidirectional cytoplasmic incompatibility

\section{Introduction}

Wolbachia are striking and intriguing maternally inherited, intracellular endosymbionts, which are probably the most widespread bacteria in invertebrates, infecting 20$76 \%$ of insect species (Jeyaprakash and Hoy, 2000; Werren and Windsor, 2000), some mites (Johanowicz and Hoy, 1995), numerous terrestrial isopods (Bouchon et $a l, 1998)$ and filarial worms (Bandi et al, 1998). Instead of increasing their hosts' reproduction or survival (Fine, 1975; Yamamura, 1993), Wolbachia have evolved toward 'reproductive parasitism', altering hosts' reproduction to facilitate their spread in the host population ( $\mathrm{O}^{\prime} \mathrm{Neill}$ et al, 1997). Examples are the feminization of genetic males in terrestrial isopods (Martin et al, 1973; Bouchon et al, 1998), parthenogenesis in Trichogramma (Stouthamer et al, 1990), male killing in Drosophila (Hurst et al, 2000) and cytoplasmic incompatibility (CI) in numerous host taxa (O'Neill et al, 1997).

The diversity of Wolbachia strains across hosts and the range of their effects cannot be explained by strict vertical transmission alone. Horizontal infectious transmission between different host species or taxa is required to explain the overall lack of congruence between host and symbiont phylogenies (Rousset et al, 1992; Rigaud and Rousset, 1996; Vavre et al, 1999).

Correspondence: C Li, Institute of Genetics, State Key Laboratory of Genetic Engineering, Fudan University, Shanghai 200433, China. E-mail: cbenli@yahoo.com

Received 21 January 2002; accepted 20 August 2002
Several studies using experimental infection have revealed that Wolbachia can infect foreign hosts. However, stable transovarial transmission of the infection in lineages after transfer has been more difficult to achieve. Failures often occurred when Wolbachia have been transfected into a host phylogenetically distant from their native host (Rigaud and Juchault, 1995; Van Meer and Stouthamer, 1999; Heath et al, 1999; Pintureau et al, 2000), whereas successes were generally found between closely related species (Boyle et al, 1993; Braig et al, 1994; Clancy and Hoffmann, 1997). Only a few attempts at horizontal transfer of Wolbachia have led to permanent establishment in a new host species (Breeuwer and Werren, 1990; Clancy and Hoffmann, 1997; Pintureau et al, 2000). The common feature of most of these experimental transfers was that either the recipients were Wolbachia negative or Wolbachia positive, but the recipients and the donors were closely related species.

Planthoppers (Homoptera: Delphacidae) are the major insect pests of rice in the world, and transmit various viral rice diseases and cause a decrease in rice yield. Among planthopper species, the small brown planthopper (Laodelphax striatellus) is one of the major vectors of rice stripe virus (RSV). In nature, some L. striatellus populations are infected with Wolbachia, and some are not (Hoshizaki, 1997; Noda et al, 2001). In this study, we reported the results of experiments that aimed to transfer the natural Wolbachia from Drosophila into L. striatellus by microinjection. Our aim was to determine if a stable superinfection could be generated and if it would result 
in the expression of CI. These experiments provide insights into the potential use of Wolbachia to spread RSV transmission blocking genes into natural populations of this insect disease vector.

\section{Materials and methods}

Wolbachia and their hosts

The following strains of Drosophila species which harbor Wolbachia infections were maintained in the laboratory: D. simulans Riverside (DSR), D. melanogaster yw67c23 (YW), D. sechellia (S9) and D. simulans Noumea (R3A). The corresponding Wolbachia were named as $w \mathrm{Ri}, w \mathrm{Mel}$, $w \mathrm{Ha}$ and $w \mathrm{No}$, respectively (provided by Prof. Scott $\mathrm{O}^{\prime}$ Neill, Queensland University, Australia). Flies were grown at $25^{\circ} \mathrm{C}$ on corn flour/sugar/yeast medium $(7.9 \mathrm{~g}$ agar, $110 \mathrm{~g}$ sucrose, $27.5 \mathrm{~g}$ yeast, $52 \mathrm{~g}$ cornmeal, $2.38 \mathrm{~g}$ Nipagin made up to 11 with water).

The small brown planthoppers (Laodelphax striatellus) were collected from Chuxiong (China) and maintained with rice seedlings, at $25^{\circ} \mathrm{C}$ in an insectary with a light cycle of $12 \mathrm{~h}$ light $/ 12 \mathrm{~h}$ dark.

\section{Wolbachia preparation and microinjection}

The Drosophila that were to serve as Wolbachia donors were washed with $70 \%$ ethanol for $5 \mathrm{~min}$, followed by three sterile water washes, $5 \mathrm{~min}$ each time. The legs of about 200 flies were removed with sterile forceps under the microscope. The flies were placed into a $0.2 \mathrm{ml}$ tube with a small hole punched in the bottom and loosely plugged with glass wool. The tube was put inside a $1.5 \mathrm{ml}$ Eppendorf tube and spun at $750 \mathrm{~g}$ for $3 \mathrm{~min}$. The hemolymph obtained was kept chilled on ice, and was then microinjected into the abdomen of $L$. striatellus within $24 \mathrm{~h}$ post-eclosion.

\section{PCR assay}

Four sets of primers were used (Zhou et al, 1998):

1. wsp $81 \mathrm{~F} / 691 \mathrm{R}$ universal for all Wolbachia strains $(81 \mathrm{~F}$ 5'-TGG TCC AAT AAG TGA TGA AGA AAC; 691R 5'-AAA AAT TAA ACG CTA CTC CA); these primers were used to amplify a DNA fragment about $610 \mathrm{bp}$

2. $w s p 202 \mathrm{~F} / 691 \mathrm{R}$ specific for L. striatellus Wolbachia (wStri) (202F 5'-AAA AGG ATA GTC CCT TAA C; 691R 5'-AAA AAT TAA ACG CTA CTC CA); these primers were used to amplify a DNA fragment of $489 \mathrm{bp}$

3. Wsp 169F/569R specific for Wolbachia from D. simulans Riverside ( $w \mathrm{Ri})$ (169F $5^{\prime}$-ATT GAA TAT AAA AAG GCC ACA GAC A; 569R 5'-CCC CCT TGT CTT TGC TTG CTG CAG), wsp 183F/570R specific for Wolbachia from $D$. simulans Noumea ( $w \mathrm{No})\left(183 \mathrm{~F} 5^{\prime}-\mathrm{AAG}\right.$ GAA CCG AAG TTC ATG; 570R 5'-GAT CTC TTT AGT AGC TGA TAC), wsp 308F/YW-R specific for Wolbachia from D. melanogaster yw67c23 (wMel) (308F 5'TTA AAG ATG TAA CAT TTG; YW-R 5'-CCG GTT GAA TTT TTA GGA TC), wsp 178F/S9-R specific for Wolbachia from $D$. sechellia $(w \mathrm{Ha})\left(178 \mathrm{~F} 55^{\prime}\right.$-AAA GAA GAC TGC GGA TAC; S9-R 5'-CCC CCT TGT CTT TGC TTG C); these primers were used to amplify a DNA fragment about $400 \mathrm{bp}$.

4. 12SAI/12SBI universal for insect mtDNA which serve as a positive control for DNA extraction (12SAI 5'-
CTA GGA TTA GAT ACC CTA TT; 12SBI 5'-AAG AGC GAC GGG GCG ATG), these primers were used to amplify a DNA fragment of approximately $400 \mathrm{bp}$.

The whole adult of Drosophila or L. striatellus minus its head was homogenized in $100 \mu \mathrm{l}$ STE $(100 \mathrm{mmol} / 1 \mathrm{NaCl}$, $10 \mathrm{mmol} / 1$ Tris- $\mathrm{HCl} \mathrm{pH} 8.0,1 \mathrm{mmol} / 1$ EDTA $\mathrm{pH} 8.0$ ) and incubated in the solution with $0.5 \mu \mathrm{g} / \mathrm{ml}$ proteinase $\mathrm{K}$ and $1 \% \mathrm{SDS}$ at $55^{\circ} \mathrm{C}$ for $1 \mathrm{~h}$. DNA was isolated by the phenol/chloroform extraction method and was finally resuspended in $30 \mu \mathrm{l}$ of $\mathrm{ddH}_{2} \mathrm{O}$. The PCR amplification was performed at 94,55 and $72{ }^{\circ} \mathrm{C}, 1 \mathrm{~min}$ each, repeated for 35 cycles in a buffer containing $2.5 \mathrm{mmol} / 1 \mathrm{MgCl}_{2}, 0.25 \mathrm{mmol} / 1 \mathrm{dNTP}$ and $500 \mathrm{nmol} / 1$ of each primer. The PCR products were analyzed by $1 \%$ agarose gel electrophoresis, while the mitochondrial primers 12SAI/12SBI were used as a positive control in a separate reaction.

Nested PCR was used to check for the existence of introduced Wolbachia. First, 81F/691R were used in PCR to check for the existence of general Wolbachia, then the PCR product was diluted 1000-fold. The specific primers for introduced Wolbachia were used for the second round of PCR. A total volume of $1 \mu \mathrm{l}$ of final PCR product was directly ligated into the vector pGEM-T (Promega) without further purification in a $10 \mu$ l-reaction overnight at $16^{\circ} \mathrm{C}$. At least three independent clones were sequenced to exclude errors introduced by Taq polymerase.

\section{Establishment of a superinfected isofemale line of L. striatellus}

After microinjection, natural male insects (1-2 individuals) were put into a culture bottle containing an injected female for mating. After about 2 weeks, larvae could be seen and the number of larvae was counted. Adult L. striatellus would emerge after 1 month. Then, one superinfected L. striatellus female and one or two males of the $F_{1}$ generation were placed into one culture bottle for mating. When the larvae of the $\mathrm{F}_{2}$ generation appeared, the $F_{1}$ generation L. striatellus were used for PCR assay to verify the existence of introduced Wolbachia. The same method was used to select the L. striatellus isofemale lines containing Drosophila-specific Wolbachia for the following generations.

\section{Determination of $\mathrm{Cl}$}

Once the L. striatellus isofemale lines with a high level of introduced Wolbachia were established, mating tests were performed to determine the presence of CI. For each species tested, the following four types of crosses were set up: superinfected female $\times$ superinfected male, superinfected female $\times$ single-infected male, single-infected female $\times$ superinfected male and single-infected female $\times$ single-infected male. All matings were set up with one female and one male (both virgins). After the offspring appeared, the parents of each cross were used to perform PCR assay for the determination of the presence of introduced Wolbachia. CI was assessed by the number of hatched larvae in each cross. In the case of multiple comparisons, one-way ANOVA and Tukey's method were used. All the related statistical analysis was done by SAS JMP software. 


\section{Results}

Detection of Wolbachia in microinjected L. striatellus lines Table 1 shows the viability rate of $L$. striatellus, the proportion that could produce offspring after being microinjected with different Wolbachia strains. The data indicates that the average viability of superinfected L. striatellus is $15.5-25.8 \%$ with $95 \%$ confidence interval. Furthermore, by Pearson's test, the probability from $\chi^{2}$ is 0.8841 , which means there was no significant difference among the different strains of Wolbachia. However, up to now we have only obtained superinfected isofemale lines of L. striatellus using $w \mathrm{Ri}$. The other three Wolbachia strains superinfected into L. striatellus were lost at the $\mathrm{F}_{1}$ generation.

The wsp gene, which is evolving at a much faster rate than any other previously reported Wolbachia genes, that is, 16S rRNA and the cell-cycle gene ftsZ (Zhou et al, 1998), was used as a probe to detect the presence of Wolbachia. To improve the sensitivity of the test, a nested PCR strategy was developed to check for the existence of introduced Wolbachia. Figure 1 shows that the injected $\mathrm{F}_{0}$ females of $L$. striatellus and some individual superinfected $L$. striatellus of the $\mathrm{F}_{1}$ generation were $w \mathrm{Ri}$ positive. The sequencing result of nested PCR product (ligated into Promega T-vector) confirmed that it was identical to the sequence of $w s p$ fragment from $w \mathrm{Ri}$, and not to the sequence of the wsp fragment from wStri that naturally infects L. striatellus.

\section{Transmission of supertransfecting Wolbachia from \\ $D$. simulans into $L$. striatellus}

Superinfected L. striatellus isofemale lines were successfully established by a one female-one male selection strategy. Table 2 shows the average frequency dynamics

Table 1 Viability rate of L. striatellus microinjected with different Wolbachia strains

\begin{tabular}{lcc}
\hline $\begin{array}{l}\text { Wolbachia } \\
\text { strain }\end{array}$ & $\begin{array}{c}\text { Number of insects } \\
\text { injected }\end{array}$ & $\begin{array}{c}\text { Number of injected insects } \\
\text { able to produce offspring }\end{array}$ \\
\hline$w \mathrm{Ri}$ & 112 & $22(19.6 \%)$ \\
$w \mathrm{No}$ & 46 & $9(19.6 \%)$ \\
$w \mathrm{Mel}$ & 54 & $13(24.1 \%)$ \\
$w \mathrm{Ha}$ & 39 & $7(17.9 \%)$ \\
Total & 251 & $51[15.5-25.8 \%]$ \\
& & (with $95 \%$ confidence interval)
\end{tabular}

$\operatorname{Prob}\left(\chi^{2}\right)=0.8841$ (Pearson test). of $w \mathrm{Ri}$ from 11 isofemale lines of superinfected L. striatellus. Figure 2 is the corresponding curve. The superinfection frequency is stable at 25.7-35.0\% (with $95 \%$ confidence intervals) in the $\mathrm{F}_{12}$ generation.

\section{Cl phenotype}

Once the L. striatellus isofemale lines with a high level of introduced Wolbachia were established, mating tests were performed to determine the presence of CI. Table 3 shows the results of test crosses for CI. Since the eggs of

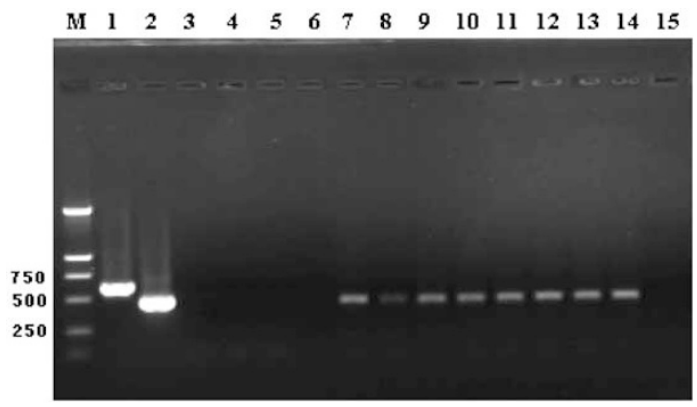

Figure 1 PCR assays of $w$ Ri infection in L. striatellus of the $\mathrm{F}_{0}$ and the $F_{1}$ generation. The results were obtained by nested PCR, amplified with $81 \mathrm{~F} / 691 \mathrm{R}$ and $169 \mathrm{~F} / 569 \mathrm{R}$. This gel shows results of second round of amplification. M: molecular weight marker (DL2000); 1: DSR amplified by universal primer 81F/691R; 2 : positive control, DSR; 3-6: negative control, L. striatellus (Chuxiong, China); 7-10: superinfected female of the $F_{0}$ generation of L. striatellus; 11-14: individuals of the $F_{1}$ generation from superinfected L. striatellus; 15: blank.

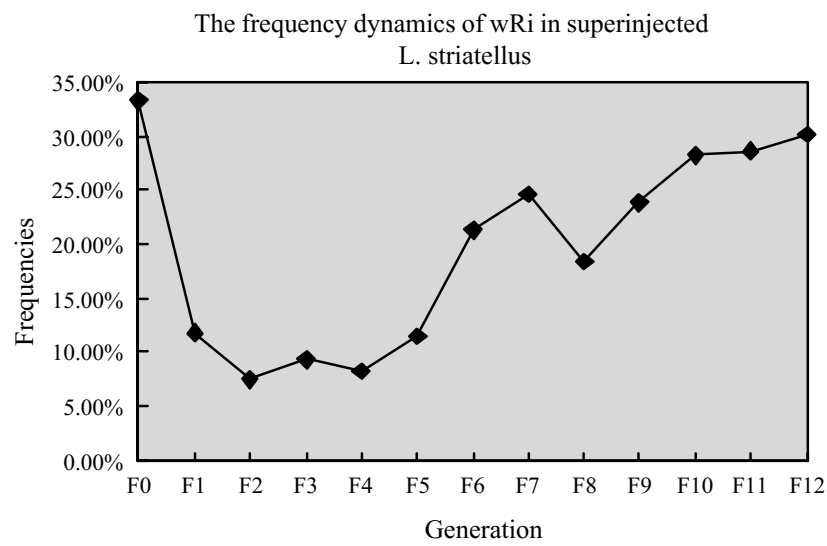

Figure 2 Frequency dynamics of $w \mathrm{Ri}$ in superinfected L. striatellus.

Table 2 Average superinfection frequency of different generations from 11 isofemale lines

\begin{tabular}{|c|c|c|c|c|c|c|c|c|c|c|c|c|c|}
\hline & $F_{0}$ & $F_{1}$ & $F_{2}$ & $F_{3}$ & $F_{4}$ & $F_{5}$ & $F_{6}$ & $F_{7}$ & $F_{8}$ & $F_{9}$ & $F_{10}$ & $F_{11}$ & $F_{12}$ \\
\hline $\mathrm{T}$ & 18 & 111 & 215 & 194 & 206 & 289 & 375 & 219 & 239 & 327 & 421 & 380 & 394 \\
\hline+ & 6 & 13 & 16 & 18 & 17 & 33 & 80 & 54 & 44 & 78 & 119 & 109 & 119 \\
\hline$\%$ & 33.3 & 11.7 & 7.4 & 9.3 & 8.3 & 11.4 & 21.3 & 24.7 & 18.4 & 23.8 & 28.3 & 28.7 & 30.2 \\
\hline 95\% UCL (\%) & 59.0 & 19.2 & 11.8 & 14.3 & 12.9 & 15.7 & 25.8 & 30.9 & 23.9 & 28.9 & 32.8 & 33.5 & 35.0 \\
\hline 95\% LCL (\%) & 13.5 & 6.4 & 4.3 & 5.6 & 4.9 & 8.0 & 17.3 & 19.1 & 13.7 & 19.3 & 24.0 & 24.2 & 25.7 \\
\hline
\end{tabular}

$\mathrm{F}_{0}-\mathrm{F}_{12}$ : generation, $\mathrm{T}$ : the total insects detected, +: the superinfected insects, \%: the superinfection frequency; UCL: upper confidence limit; LCL: lower confidence limit. 


\begin{tabular}{|c|c|c|c|c|}
\hline & $\begin{array}{c}B \\
q++\times \widehat{\delta}++\end{array}$ & $\underset{q++\times \delta+}{C}$ & $\begin{array}{c}D \\
q+\times \delta_{++}\end{array}$ & $\begin{array}{c}E \\
q+\times ð+\end{array}$ \\
\hline Average number of offspring & 55.6 & 56.5 & 3.1 & 60 \\
\hline (No. of crosses) & (10) & $(10)$ & $(10)$ & $(10)$ \\
\hline SD & 11.0 & 8.7 & 5.4 & 14.0 \\
\hline $95 \%$ UCL & 63.5 & 61.5 & 5.8 & 70.0 \\
\hline $95 \% \mathrm{LCL}$ & 47.7 & 49.4 & 0 & 50.0 \\
\hline
\end{tabular}

UCL: upper confidence limit, LCL: lower confidence limit; ++: superinfection; +: single infection.

Table 4 Average numbers of offspring in each generation from superinfected L.striatellus

\begin{tabular}{lccc}
\hline & $\begin{array}{c}\text { Average number } \\
\text { of offspring }\end{array}$ & SD & 95\% confidence value \\
\hline $\mathrm{F}_{1}(10)$ & 25.2 & 24.7 & \\
$\mathrm{~F}_{2}(35)$ & 26.4 & 18.5 & 15.3 \\
$\mathrm{~F}_{3}(60)$ & 15.6 & 18.2 & 6.1 \\
$\mathrm{~F}_{4}(74)$ & 17.5 & 18.6 & 4.6 \\
$\mathrm{~F}_{5}(29)$ & 17.6 & 18.0 & 6.3 \\
$\mathrm{~F}_{6}(55)$ & 22.5 & 18.1 & 4.8 \\
$\mathrm{~F}_{7}(25)$ & 20.6 & 17.0 & 6.5 \\
$\mathrm{~F}_{8}(27)$ & 35.8 & 24.1 & 9.1 \\
$\mathrm{~F}_{9}(30)$ & 30.0 & 21.2 & 7.6 \\
$\mathrm{~F}_{10}(65)$ & 35.8 & 23.6 & 5.7 \\
$\mathrm{~F}_{11}(33)$ & 50.1 & 19.8 & 6.7 \\
\hline
\end{tabular}

The number in brackets means the number of crosses analyzed.

L. striatellus were wrapped in the leaves of seedlings, the growth of superinfected L. striatellus would obviously be affected if the eggs were cultured outside the seedlings. So the number of nymphs of each cross was used as the parameter for the CI test instead. Our original hypothesis is: $\mathrm{H}_{\mathrm{o}}: \mu_{\mathrm{B}}=\mu_{\mathrm{C}}=\mu_{\mathrm{D}}=\mu_{\mathrm{E}}$; the alternative hypothesis is $\mathrm{H}_{\mathrm{a}}$ : not all $\mu_{i}$ are equal $(i=\mathrm{B}, \mathrm{C}, \mathrm{D}, \mathrm{E})$. Prob. based on the statistic $=0.0001$ was obtained by one-way ANOVA, which means that there is a significant difference among the four types of the crosses. Further by using Tukey's method, Cross D is significantly different from the other three crosses, while there are no significant differences among Crosses B, C and E.

Results from the statistical analysis showed that the superinfected L. striatellus males displayed high percentages of unidirectional CI when crossed with the naturally single-infected females, while the superinfected females were compatible with both superinfected males and single-infected males.

\section{Wolbachia segregation from superinfected $L$. striatellus} According to the results of nested PCR analysis, the products of the first round of PCR from some offsprings of superinfected L. striatellus were positive, whereas those of the final round of PCR were positive or negative. Some superinfected isofemale lines even lost their superinfection characteristics during the selection process. It indicated the existence of heterogeneity of offspring of the superinfected L. striatellus.
Relationship between the introduced Wolbachia and superinfected $L$. striatellus

Considering that the average fecundity of superinfected L. striatellus increased with each successive generation (Table 4), up to the $F_{11}$ generation, the average fecundity is approximately normal (results from CI test). This suggests that the local adaptation between introduced Wolbachia and the new host has been established, from a low level of 'disruption' to 'compatibility'.

\section{Discussion}

\section{Establishment of superinfected $L$. striatellus isofemale lines}

This study has shown that Wolbachia can be transferred between phylogenetically distant insects, and that the superinfection is inherited to the $F_{12}$ generation. The spread of superinfected Wolbachia within a new host population requires stable vertical transmission. However, the establishment of stable vertical transmission within new hosts requires the following conditions.

The first concerns the Wolbachia strains. To address the potential effects of Wolbachia strains on superinfection, a comparison was made by superinfecting different Wolbachia strains into the same host background. As shown by experimental data, only $w$ Ri were successfully superinfected into the naturally single-infected L. striatellus. This is supported by the fact that $w \mathrm{Ri}$ are more phylogenetically distant from $w$ Stri than are $w \mathrm{Ha}$ and $w$ No (Zhou et al, 1998). On the another hand, although $w \mathrm{Mel}$ are also more phylogenetically distant from $w$ Stri than $w \mathrm{Ri}$, the CI phenotype of $w \mathrm{Mel}$ is weaker than that of $w \mathrm{Ri}$. Moreover, compared to the infection of different Wolbachia strains, wRi infection may be much more recent, because they are associated with a specific mitochondrial DNA subtype (Hale and Hoffmann, 1990; Turelli et al, 1992), and it is easier for $w \mathrm{Ri}$ to adapt to the physiological environment of the novel host. All the above indicates that $w \mathrm{Ri}$, phylogenetically distant from the naturally existing Wolbachia, with the ability to induce strong CI, will play an important role in the route of superinfection.

The second point concerns the relationship between introduced Wolbachia and their new hosts. In order to be established in a new host population after infection, Wolbachia symbionts have three requirements: (i) compatibility; (ii) transmission, the ability for the infection to be transmitted in the novel host; and (iii) disruption, the ability to turn the host's reproduction to their own advantage, thereby creating a mechanism for spreading 
in the new host population. The results from Table 4 are consistent with the progression from a small amount of 'disruption' to 'compatibility', demonstrating that it takes a long time for introduced Wolbachia to establish a higher infection frequency in the L. striatellus population. However, knowledge of the detailed mechanisms of interaction between Wolbachia and the new host requires further study.

Our third point concerns the vertical transmission of the introduced Wolbachia. For vertical transmission, Wolbachia must be present in mature and viable eggs, which requires that they undergo replication and segregation synchronous with their host during oogenesis. Our results are compatible with the lack of congruence found between molecular phylogenies of parasitoid guilds and their hosts (Plantard et al, 1998; West et al, 1998). This raises the possibility that it is the vertical component of transmission, not the horizontal one, that is one of the rate-limiting steps in the spread and maintenance of Wolbachia in new host populations. In our study, to address this problem, hemolymph of Drosophila was microinjected into the abdomen of L. striatellus. The aim of this method is to strengthen the vertical transmission of Wolbachia. One reason is that hemolymph offers a similar physiological environment between the recipients and donors. Secondly, it would benefit the vertical transmission of Wolbachia since the abdomen contains the reproductive tissues.

Moreover, theoretical arguments suggest that the initial infection frequencies may influence whether or not the infection ultimately becomes evolutionarily stable (Rigaud et al, 2001). The existence of Wolbachia segregation from superinfected L. striatellus indicated that low densities of introduced Wolbachia in the host insect would appear to produce a proportion of uninfected cystoblasts as a result of the stochastic loss of bacteria during mitosis, thus jeopardizing successful vertical transmission of the superinfection. A very high level of introduced Wolbachia infection in the donor hosts, specific aspects of the introduced Wolbachia's development, or a combination of the two factors may have important influences on the initial titer of Wolbachia.

In conclusion, successful horizontal transmission of Wolbachia between phylogenetically distantly related species would probably require strong selection in a new Wolbachia variant. This does not mean that such transfers are impossible, as suggested by phylogenetic analyses (Bouchon et al, 1998; Cordaux et al, 2001), but these events will be rare. From the point of view of evolution, maybe several million years ago Wolbachia had more potential (plasticity) to infect several host species than at present, and they may have lost this following selection and coevolution with particular hosts. There is a possibility, however, that some Wolbachia lineages are less specialized and could infect other host species more easily, that is, wRi in our study.

\section{Superinfected L. striatellus could take advantage of unidirectional $\mathrm{Cl}$ to spread in their natural population} The superinfected males of L. striatellus showed strong unidirectional CI with the naturally single-infected female of L. striatellus. As a result, superinfected females have a reproductive advantage in a mixed population of infected and uninfected individuals (Caspari and Watson, 1959). Since Wolbachia are maternally inherited, this process of unidirectional incompatibility will lead to a rapid increase in the proportion of infected hosts in an interbreeding population (Turelli and Hoffmann, 1991). The results from our experiments indicate that a higher density of introduced Wolbachia could cause stronger CI, while at the same time CI itself strengthens the spread of introduced Wolbachia in the whole population. These two factors interact with each other to spread the introduced Wolbachia in the new host.

It should be noted that the induction of CI by $w \mathrm{Ri}$ in superinfected L. striatellus was even stronger than in $D$. simulans (Turelli and Hoffmann, 1995). The reason behind this is still unknown. The effects of host, the naturally existing Wolbachia, or a combination of the two factors, might be responsible for it.

\section{Conclusion}

Our study provides the possibility of supertransfecting CI-causing Wolbachia into L. striatellus from a phylogenetically distant host by adult female microinjection. The introduced Wolbachia also induce the same CI phenotype as in the former hosts. The superinfected L. striatellus show unidirectional CI when mating with the original naturally infected species, which means the superinfected $L$. striatellus have a reproductive advantage in the natural population. Our results provide insights into the potential use of Wolbachia to spread RSV transmission blocking genes into natural populations of this insect disease vector.

\section{Acknowledgements}

We are grateful to Professor Scott L O'Neill, Prof. Thomas A Miller, Dr Jian Yan and Dr Xiaohui Wu for helpful comments on the manuscript. We would like to thank Ms. Rong Ma for the help on statistics, Dr Weiguo Zhou, Prof. Deming Su, Prof. Jiang Zhong, Dr Zhicai $\mathrm{Qu}$ and Ms Beibei Ying for helpful discussion. This work was supported by McKnight Foundation, USA, and by National Science Foundation, China (39800085).

\section{References}

Bandi C, Anderson TJC, Genshi C, Blaxter ML (1998). Phylogeny of Wolbachia in filarial nematodes. Proc $R$ Soc London B 265: 2407-2413.

Bouchon D, Rigaud T, Juchault P (1998). Evidence for widespread Wolbachia infection in isopod crustaceans: molecular identification and host feminization. Proc $R$ Soc London B 265: 1081-1090.

Boyle L, O'Neill SL, Robertson HM, Karr TL (1993). Interspecific and intraspecific horizontal transfer of Wolbachia in Drosophila. Science 260: 1796-1799.

Braig HR, Guzman H, Tesh RB, O’ Neill SL (1994). Replacement of the natural Wolbachia symbiont of Drosophila simulans with a mosquito counterpart. Nature 367: 453-455.

Breeuwer JA, Werren JH (1990). Microorganisms associated with chromosome destruction and reproductive isolation between two insect species. Nature 346: 558-560.

Caspari E, Watson GS (1959). On the evolutionary importance of cytoplasmic sterility in mosquitoes. Evolution 13: 568-570. 
Clancy DJ, Hoffmann AA (1997). Behavior of Wolbachia endosymbionts from Drosophila simulans in D. serrata, a novel host. Am Nat 149: 975-988.

Cordaux R, Michel-Salzat A, Bouchon D. (2001). Wolbachia infection in crustaceans: novel hosts and potential routes for horizontal transmission. J Evol Biol 14: 237-243.

Fine PEM (1975). Vectors and vertical transmission: an epidemiologic perspective. Ann N Y Acad Sci 266: 173-194.

Hale LR, Hoffmann AA (1990). Mitochondrial DNA polymorphism and cytoplasmic incompatibility in natural population of Drosophila simulans. Evolution 44: 1383-1386.

Heath BD, Butcher RDJ, Whitfield WGF, Hubbard SF (1999). Horizontal transfer of Wolbachia between phylogenetically distant insect species by a natural occurring mechanism. Curr Biol 9: 313-316.

Hoshizaki S (1997). Allozyme polymorphism and geographic variation in the small brown planthopper, Laodelphax striatellus (Homoptera: Delphacidae). Biochem Genet 35: 383-393.

Hurst GD, Johnson AP, Schulenburg JH, Fuyama Y (2000). Male-killing Wolbachia in Drosophila: a temperature-sensitive trait with a threshold bacterial density. Genetics 156: 699-709.

Jeyaprakash A, Hoy MA (2000). Long PCR improves Wolbachia DNA amplification: wsp sequences found in $76 \%$ of sixtythree arthropod species. Insect Mol Biol 9: 393-405.

Johanowicz DL, Hoy MA (1995). Molecular evidence for AWolbachia endosymbiont in the predatory mite Metaseiulus accidentalis. I Cell Biochem 21A: 198.

Martin G, Juchault P, Legrand JJ (1973). Mise en e' videnced'un micro-organisme intracytoplasmique symbiote de l'oniscoide Armadillidium vulgare (L.), dont la pre' sence accompagnel'intersexualite' ou la fe' minisation totale des mâ les ge'ne' tiques de la ligne' e the' lyge' ne. C R Acad Sci Paris III 276: 2313-2316.

Noda H, Koizumi Y, Zhang Q, Deng K (2001). Infection density of Wolbachia and incompatibility level in two planthopper species, Laodelphax striatellus and Sogatella furcifera. Insect Biochem Mol Biol 31: 727-737.

O'Neill SL, Hoffmann AA, Werren JH (1997). Influential Passengers: Inherited Microorganisms and Arthropod Reproduction. Oxford University Press, Oxford.

Pintureau B, Grenier S, Bole' at B, Lassablie're F, Heddi A, Khatchadourian C (2000). Dynamics of Wolbachia populations in transfected lines of Trichogramma. J Invertebr Pathol 76 20-25.

Plantard O, Rasplus JY, Mondor G, LeClaainche I, Solignac M (1998). Wolbachia-induced thelytoky in the rose gallwasp
Diplolepis spinosissimae(Giraud) (Hymenoptera: Cynipidae), and its consequences on the genetic structure if its host. Proc $R$ Soc London B 265: 1075-1080.

Rigaud T, Pennings PS, Juchault P (2001). Wolbachia bacteria effects after experimental interspecific transfers in terrestrial isopods. J Invertebr Pathol 77: 251-257.

Rigaud T, Juchault P (1995). Success and failure of horizontal transfers of feminizing Wolbachia endosymbionts in woodlice. J Evol Biol 8: 249-255.

Rigaud T, Rousset F (1996). What generates the diversity of Wolbachia-arthropod interactions? Biodivers Conserv 5: 999_ 1013.

Rousset F, Bouchon D, Pintureau B, Juchault P, Solignac M (1992). Wolbachia endosymbionts responsible for various alterations of sexuality in arthropods. Proc $R$ Soc London $B$ 250: 91-98.

Stouthamer R, Luck RF, Hamilton WD (1990). Antibiotics cause parthenogenetic Trichogramma (Hymenoptera/Trichogrammatidae) to revert to sex. Proc Natl Acad Sci USA 87: 24242427.

Turelli M, Hoffmann AA (1991). Rapid spread of an inherited incompatibility factor in California Drosophila. Nature 353: 440-442.

Turelli M, Hoffmann AA (1995). Cytoplasmic incompatibility in Drosophila simulans: dynamics and parameter estimates from natural population. Genetics 140: 1319-1338.

Turelli M, Hoffmannn AA, Mckechnie SW (1992). Dynamics of cytoplasmic incompatibility and mtDNA variation in natural Drosophila simulans populations. Genetics 132: 713-723.

Van Meer MM, Stouthamer R (1999). Cross-order transfer of Wolbachia from Muscidifurax uniraptor (Hymenoptera: Pteromalidae) to Drosophila simulans (Diptera: Drosophilidae). Heredity 82:163-169.

Vavre F, Fleury F, Lepetit D, Fouillet P, Boule' treau M (1999). Phylogenetic evidence for horizontal transmission of Wolbachia in host-parasitoid associations. Mol Biol Evol 16: 1711-1723.

Werren JH, Windsor D (2000). Wolbachia infection frequencies in insects: evidence of a global equilibrium. Proc $R$ Soc London $B$ 267: 1277-1285.

West SA, Cook JM, Werren JH, Godfray HCJ (1998). Wolbachia in the two insect host-parasitoid communities. Mol Ecol 7: 14571465.

Yamamura N (1993). Vertical transmission and evolution of mutualism for parasitism. Theor Pop Biol 44: 95-109.

Zhou WG, Rousset F, O'Neill SL (1998). Phylogeny and PCR-based classification of Wolbachia strains using wsp gene sequences. Proc R Soc London 265: 509-515. 